\title{
Improving the Resolution of Non-Invasive Time Domain Reflectometry
}

\author{
Ian G. Platt and Ian M. Woodhead \\ Lincoln Ventures Ltd, \\ Christchurch, New Zealand \\ platti@lvl.co.nz
}

\begin{abstract}
Non Invasive Time Domain Reflectometry (TDR) may be used to estimate the volumetric moisture content, $\theta_{v}$, with depth for a variety of sample materials. The forward physical model is couched in terms of a moments method where integration is performed over a discretised sample space to estimate the measured propagation time, $t_{p}$ down a pair of parallel transmission lines. We show that inverse solution to this, which recovers relative permittivity and thus $\theta_{v}$, is greatly facilitated by a simplification of the system geometry via, 1) realistically modeling the prior density of the sample, 2) using this prior with the inherent system symmetry to reduce the number of required discretisation cells, and 3) determining a physically meaningful reduction operator to allow a coarse discretisation mesh to be used. The observational equation is expressed in the Bayesian paradigm with the most accurate and robust solution obtained using the Conditional Mean of the posterior distribution constructed via a Monte Carlo method. Results of simulation show that the method is capable of providing accurate estimates of the moisture density profile down to a depth of $100 \mathrm{~mm}$ with an error $<4 \%$. Further, the reduction in the number of discretised cells required to accurately estimate these profiles means that the inversion procedure is quick enough to enable the real time application of the equipment, a fundamental requirement in the development.
\end{abstract}

Keywords: Time domain reflectometry, Bayesian, Non invasive, Moisture content, Transmission lines, Electromagnetic sensor.

\section{Introduction}

The ability to calculate moisture content in soils has been developed over the past 50 years, primarily to maximize water use efficiency in agriculture and to understand subsurface hydrological processes. Three basic methods have been developed over this period, namely; gravimetric, neutron scattering and electromagnetic [1] [2]. Gravimetric methods are accurate but have the disadvantage that the sample must be removed, while neutron scattering sensors must be closely monitored and cannot be left unattended, since they pose a health risk. The role of electromagnetic sensors, which suffers neither of these disadvantages, has thus become of increasing importance for soil moisture content measurement. Most of the current electromagnetic systems estimate the relative permittivity, $\epsilon_{r}$, of the sample by deploying two or more metallic probes into the soil to measure the capacitance or impedance between between them, or as in the case Time Domain Reflectometry (TDR), the time delay of a pulse sent down a pair of probes acting as a transmission line. The $\epsilon_{r}$ of the sample is then related to its volumetric moisture content, $\theta_{v}$, by an empirical relationship such as those derived by [3] and [4]. Since water has a high relative permittivity, $\epsilon_{r} \sim 80$, compared to values of $\epsilon_{r} \leq 5$ for most other components that make up typical samples the pulses velocity change is most sensitive to the amount of water present. The measured permittivity can be related to the samples dielectric properties by a real part representing the stored energy of the system and an imaginary part describing its energy dissipation:

$$
\epsilon_{r}=\stackrel{\prime}{\epsilon}_{r}-\jmath\left(\stackrel{\prime \prime}{\epsilon}_{\text {relax }}+\frac{\sigma_{d c}}{2 \pi f \epsilon_{0}}\right)
$$

\section{Where}

$\stackrel{1}{\epsilon}_{r} \quad$ is the stored energy of the system

$\sigma_{d c} \quad$ the electrical conductivity between the probes.

" $\epsilon_{\text {relax }}$ is the molecular relaxation of water molecules

$t$

is the frequency of the probe signal.

In this work we assume a lossless media so that the imaginary part of Equation 1 becomes negligible and the effective permittivity for the system is $\epsilon_{r}^{\prime}$. In those cases where this is not justifiable [5] have developed a method whereby allowance for the conductivity part of the loss can be easily incorporated.

For the invasive TDR system a pair of parallel transmission lines are inserted into the sample and a short pulse is sent down them generating a static electric field. This field causes the sample dielectric to produce an opposing polarization field that reduces the phase velocity of the pulse. The velocity of the pulse can be given in terms of the permittivity and permeability of the sample by:

$$
v_{p}=\frac{c}{\sqrt{\epsilon_{r} \mu_{r}}}
$$

where $\epsilon_{r}=\epsilon / \epsilon_{0}$ is the relative permittivity, $c$ is the 
speed of light and $\mu_{r}$ is the relative permeability (which is equal to one for most soils). By measuring the time the pulse takes to travel down the transmission line of length, $d$, and back again after a reflection from the end, the propagation time can be related to $\epsilon_{r}$ from Equation 2 (with $\mu=1$ ) by:

$$
t_{p}=\frac{2 d}{c} \sqrt{\epsilon_{r}}
$$

Woodhead [6] extended this TDR arrangement by deploying the transmission lines external to the sample, effectively creating a non-invasive system. Being noninvasive the system can be used on a greater variety of materials including timber, concrete, road seal etc, with the added advantage that it does not alter the medium (.e.g. cause water wells around inserted probes) being tested. For this new system geometry the relationship defined by Equations 2 and 3 no longer apply. Using a tomographic technique based upon discretisation of the $\mathrm{x}-\mathrm{y}$ plane through the system geometry (Figure 1), a new equation relating the pulse propagation time, $t_{p}$, to the sample permittivity, $\epsilon_{r}$, can be constructed [6] of the form:

$$
t_{p}=f\left(\epsilon_{r}\right)
$$

This equation represents the forward construction of the physical system where the form of $f$ is specified in Section 2. Since we seek an estimate of $\epsilon_{r}$ from a measured value of $t_{p}$ it is the inverse of this equation that is required:

$$
\epsilon_{r}=f^{-1}\left(t_{p}\right)
$$

In common with many other inverse problems, the solution of Equation 5 is somewhat problematic. The forward function is both non-linear and ill-conditioned thus an estimate of $\epsilon_{r}$ by the inverse process requires some form of regularized optimization technique. Further, since no analytic solution to the forward model is available, the method of moments technique in which the sample is discretised, is used as an approximation to the solution. The level of discretisation, or mesh size, will of course have an effect upon the accuracy of this approximation.

The objective of this paper is to describe a methodology for improving the accuracy and efficiency of the inverse defined by Equation 5 via a Monte Carlo simulation.

\section{Background}

The steps in defining the function, $f$ in the forward model of Equation 4 are derived by Woodhead [6] which we briefly recount here. When an incident electric field, $\mathbf{E}_{\mathbf{i}}$ is imposed upon a dielectric of volume $\tau$, the dipole moment $\mathbf{P}$, of the volume produces an electric field, $\mathbf{E}_{\mathbf{p}}$. The total electric field is then:

$$
\mathbf{E}_{\mathbf{t}}=\mathbf{E}_{\mathbf{i}}+\mathbf{E}_{\mathbf{p}}
$$

Using the constitutive relationship $E \epsilon_{0}\left(\epsilon_{r}-1\right)=P$ this becomes, after some re-arrangement:

$$
-\mathbf{E}_{\mathbf{i}}=\mathbf{E}_{\mathbf{p}}-\frac{\mathbf{P}}{\epsilon_{0}\left(\epsilon_{\mathbf{r}}-1\right)}
$$

Where $\epsilon_{0}$ is the permittivity of free space and $\epsilon_{\mathbf{r}}$ is the relative permittivity of the dielectric. $\mathbf{E}_{\mathbf{p}}$ may be calculated at a point $\mathbf{r}$ from the polarization element $\mathbf{P}$ via:

$$
\mathbf{E}_{\mathbf{p}}=-\int_{\tau} \nabla\left(\frac{1}{4 \pi \epsilon_{0}} \frac{\mathbf{P} \cdot \mathbf{r}}{r^{3}}\right) d \tau
$$

so that Equation 7 becomes:

$$
-\mathbf{E}_{\mathbf{i}}=-\int_{\tau} \nabla\left(\frac{1}{4 \pi \epsilon_{0}} \frac{\mathbf{P} \cdot \mathbf{r}}{r^{3}}\right) d \tau-\frac{\mathbf{P}}{\epsilon_{0}\left(\epsilon_{\mathbf{r}}-1\right)}
$$

and this may be expressed in terms of a linear operator, $\mathbf{L}$, acting upon $\mathbf{P}$, [10] as:

$$
-\mathbf{E}_{\mathbf{i}}=\mathbf{L}(\mathbf{P})
$$

Upon discretisation into cells into $N$ cells, as depicted in Figure 1, the polarization field of Equation 8 becomes:

$$
\mathbf{E}_{\mathbf{p}_{m}}=-\nabla\left(\sum_{n=1}^{N} \frac{1}{4 \pi \epsilon_{0}} \frac{\mathbf{P} \cdot \mathbf{r}}{r^{3}}\right) \Delta \tau
$$

Where $m$ is the cell at which the polarization field from cell $n$ (source cell) is to be calculated. Since, by model assumption, the z-component is constant, expanding over the gradient function gives:

$$
\begin{aligned}
\mathbf{E}_{\mathbf{p}_{m}}=\sum_{n=1}^{N} \frac{1}{4 \pi \epsilon_{0}} \\
\left.\qquad \begin{array}{cc}
\frac{3 x_{m n}^{2}-r_{m n}^{2}}{r_{m n}^{5}} & \frac{3 x_{m n} y_{m n}}{r_{m n}^{5}} \\
\frac{3 x_{m n} y_{m n}}{r_{m n}^{5}} & \frac{3 y_{m n}^{2}-r_{m n}^{2}}{r_{m n}^{5}}
\end{array}\right) \mathbf{P}_{\mathbf{n}}
\end{aligned}
$$


Where $x_{m n}$ and $y_{m n}$ are the $x$ and $y$ differences between the points $m$ and $n$ respectively. Note that when $m=n$ (i.e the cells own polarization field) components become:

$$
\mathbf{E}_{\mathbf{p}_{m}}=\frac{1}{4 \pi \epsilon_{0}}\left[\left(\begin{array}{cc}
\frac{1}{3 \epsilon_{0}} & 0 \\
0 & \frac{1}{3 \epsilon_{0}}
\end{array}\right)\right] \mathbf{P}_{\mathbf{m}}
$$

By using the method of moments with a subsectional basis function defined as non zero only for the source cell, [10] showed that Equation 10 can be written in the discrete matrix form:

$$
-\mathbf{E}_{\mathbf{i}}=\mathbf{L} \mathbf{P}
$$

with the components of $\mathbf{L}$ given by the square bracketed part of Equation 12 for $m \neq n$ and by:

$$
\frac{1}{4 \pi \epsilon_{0}}\left[\left(\begin{array}{cc}
\frac{1}{3 \epsilon_{0}} & 0 \\
0 & \frac{1}{3 \epsilon_{0}}
\end{array}\right)\right]+\frac{1}{\Delta \epsilon}
$$

when $m=n$, where $\Delta \epsilon=\epsilon_{0}\left(\epsilon_{r}-1\right)$. Since both $\mathbf{L}$ and $\mathbf{P}$ are functions of the independent variable $\epsilon_{\mathbf{r}}$, Equation 14 is non-linear.

Woodhead [6] extended the discretised model of Equation 14 by introducing the concept of a transmission line to produce the incident field $\mathbf{E}_{\mathbf{i}}$, so that the pulse velocity, $v$, due to the dielectric interaction can be given by the telegraphers equation (e.g. [11]):

$$
v=\frac{2 d}{t_{p}}=\sqrt{\frac{\pi V_{t}}{\rho \mu \cosh ^{-1}\left(\frac{b}{a}\right)}}
$$

so that

$$
t_{p}=2 d\left(\frac{\pi V_{t}}{\rho \mu \cosh ^{-1}\left(\frac{b}{a}\right)}\right)^{-1 / 2}
$$

Where
$V_{t}=V_{i}+V_{p}$ is the total voltage between the transmission lines, $V_{p}$ is the component due to the polarization field induced by the dielectric sample and $V_{i}$ is the component due to the incident electric field directly from the transmission lines.

$d \quad$ is the length of the transmission line.

$t_{p}$ is the propagation time of the pulse from the beginning of the line to its reflection at the end and back to the beginning.

$\rho \quad$ is the line charge density.

$\mu \quad$ is the magnetic permeability.

$b \quad$ is the spacing between the parallel lines, and

$a$ is the diameter of the transmission lines.

The only unknown on the right hand side of this equation is the polarization induced voltage, $V_{p}$, so that this equation may be succinctly represented by:

$$
t_{p}=\Psi\left(V_{p}\right)
$$

Following the method of [7] both the transmission lines and sample can be imbedded in the discretisation space, depicted by Figure 1, so that:

$$
V_{p}=w \sum_{m=1}^{M} \mathbf{E}_{\mathbf{p}_{\mathbf{m}}}
$$

where $\mathrm{M}$ are the number of cells between the transmission lines and $w$ is the width of the cell.

For any particular value of permittivity in each sample cell, the polarization $\mathbf{P}$ within it can be calculated from Equation 14 since $\mathbf{P}=-\mathbf{L}^{-1} \mathbf{E}_{\mathbf{i}}$, so for those cells:

$$
\begin{aligned}
V_{p} & =w \sum_{m=1}^{M} \mathbf{E}_{\mathbf{p}_{\mathbf{m}}} \\
& =w \sum_{m=1}^{M} \frac{1}{\Delta \epsilon} \mathbf{P}_{\mathbf{n}} \delta_{\mathrm{mn}} \\
& =w \sum_{m=1}^{M} \frac{1}{\Delta \epsilon}\left(\mathbf{L}^{-\mathbf{1}} \mathbf{E}_{\mathbf{i}}\right)_{n} \delta_{\mathrm{mn}}
\end{aligned}
$$

Where the delta function indicates that only components of cells between the transmission lines are selected. We represent this in the simplifying function notation as:

$$
V_{p}=\Phi\left(\epsilon_{r}\right)
$$


The forward solution defined by Equation 4 can now be formed by the composition of the two functions $\Psi$ and $\Phi$ so that:

$$
f\left(\epsilon_{r}\right)=\Psi\left(\Phi\left(\epsilon_{r}\right)\right)
$$

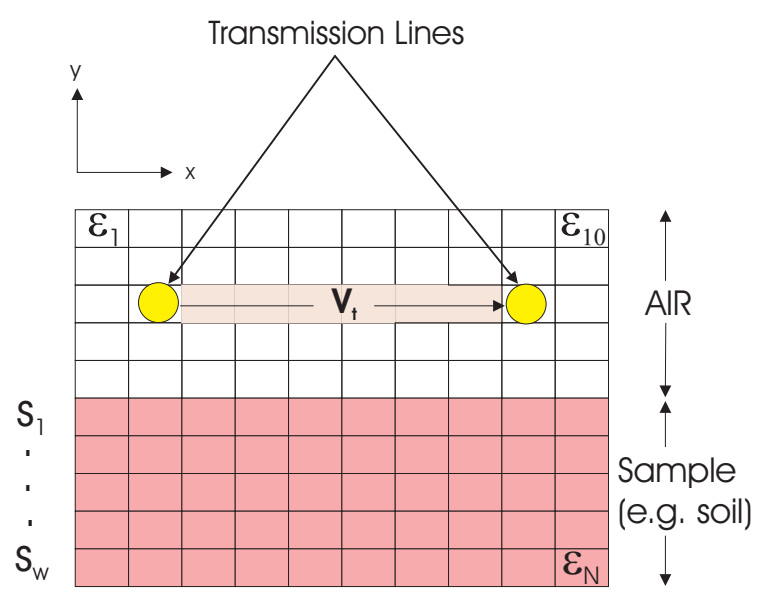

Figure 1: The 2D geometry of discrete cells and parallel transmission lines used to define the system matrix equation. The cross hatched cells between the transmission lines are those ( $\mathrm{M}$ of them) included in the calculation of $\mathrm{E}_{t}$. Each of the cells has a width w.

\section{Bayesian Formulation}

In exploring the solution to the inverse problem outlined by Equation 4 we use the Bayesian framework as described for example by [8] and [9]. In this representation Equation 4 may be written in the observational form as:

$$
T=F\left(\epsilon_{r}\right)+\mathcal{E}_{n}
$$

Where now $T$ and $F$ represent the random variables (with $t_{p}$ and $f\left(\epsilon_{r}\right)$ as realizations) and we have added $\mathcal{E}_{n}$ as the measurement noise, assumed to be independent of $F$. An inverse solution to this equation can be given in terms of the variable densities by the well know Bayes Formula:

$$
\pi\left(\epsilon_{r} \mid t_{p}\right)=\frac{\pi\left(t_{p} \mid \epsilon_{r}\right) \pi_{\mathrm{pr}}\left(\epsilon_{r}\right)}{\pi\left(t_{p}\right)}
$$

Where $\pi\left(\epsilon_{r} \mid t_{p}\right)$ is the posterior density, $\pi\left(t_{p} \mid \epsilon_{r}\right)$ is the likelihood density function and $\pi_{p r}\left(\epsilon_{r}\right)$ is the prior density function of the permittivity and will act as a regularization for different physical assumptions about the likely structural disposition of $\epsilon_{r}$ throughout the sample. The term $\pi\left(t_{p}\right)$, often called the evidence factor, can be viewed as a scaling factor that ensures a unit total probability of the posterior density function. Since it plays no part in the evaluation of the required parameters of the posterior distribution it is usually ignored so that the above equation becomes:

$$
\pi\left(\epsilon_{r} \mid t_{p}\right) \propto \pi\left(t_{p} \mid \epsilon_{r}\right) \pi_{\mathrm{pr}}\left(\epsilon_{r}\right)
$$

To obtain an estimate of $\epsilon_{r}$ corresponding to the measurement $t_{p}$ we can use either the Maximum A Posteriori Estimator (MAP) or Conditional Mean (CM) of the posterior distribution given by Equation 25, with associated errors given by the covariance $\Gamma_{\epsilon}$. Both the $\mathrm{CM}$ and $\Gamma_{\epsilon}$ estimates require integration over the posterior density $\pi\left(\epsilon_{r} \mid t_{p}\right)$ :

$$
\epsilon_{\mathrm{CM}}=\int \epsilon_{r} \pi\left(\epsilon_{r} \mid t_{p}\right) d \epsilon_{r}
$$

with the estimate error given by the posterior covariance

$$
\begin{aligned}
\Gamma_{\epsilon} & =\operatorname{cov}\left(\epsilon_{r} \mid t_{p}\right) \\
& =\int\left(\epsilon_{r}-\epsilon_{\mathrm{CM}}\right)\left(\epsilon_{r}-\epsilon_{\mathrm{CM}}\right)^{T} \pi\left(\epsilon_{r} \mid t_{p}\right) d \epsilon_{r}
\end{aligned}
$$

while the MAP estimate:

$$
\epsilon_{\mathrm{MAP}}=\arg \max \pi\left(\epsilon_{r} \mid t_{p}\right)
$$

is usually achieved by some sort of optimization method.

The construction of the applicable form of Equation 25 for the TDR model will be detailed in Section 3.4 after some relevant properties of the system (such as parameter independence) are established.

\subsection{Required Level of Discretisation}

In the observational model of Equation 23, $T$ is the measured value of the propagation time, with realization $T=t_{p}$. Since no measurements are available, model validation must be carried out using simulated data which should be generated at a high enough level of resolution that it is as close as possible to the perceived real case. For this case we content ourselves with a level of discretisation that provides a model accuracy that cannot be much improved upon by further decreases in mesh size. To determine this level of discretisation, the polarization electric field, $\mathbf{E}_{\mathbf{p}}$, between the transmission lines was calculated using Equation 22 for an increasing number of individual cells over a constant sample volume. The results of this are shown in Figure 2. Here the polarization field converges to that given by the mesh sizes of Res 4 - 5 (defined in Table 1). 
Table 1: Discretisation values. $n_{x}, n_{y}, n_{z}$ are the number of cells in the directions $\mathrm{x}, \mathrm{y}$ and $\mathrm{z}$ respectively.

\begin{tabular}{|l|llll|}
\hline $\begin{array}{l}\text { Resolution } \\
\text { number }\end{array}$ & Cell Size (m) & $n_{x}$ & $n_{y}$ & $n_{z}$ \\
\hline Res 1 & 0.100 & 4 & 1 & 1 \\
Res 2 & 0.033 & 12 & 3 & 3 \\
Res 3 & 0.020 & 20 & 5 & 5 \\
Res 4 & 0.014 & 28 & 7 & 7 \\
Res 5 & 0.011 & 36 & 9 & 9 \\
\hline
\end{tabular}

Since Res 5 is computationally inaccessible, even for the small number of test cases required in the current development, Res 4 is chosen as being close enough to the continuous case to well approximate the operational system. Computationally it is only accessible for a limited number of calculations will be used in section 3.4 to construct a resolution reduction operator $q$ and in section 3.4 to test the inversion procedure.

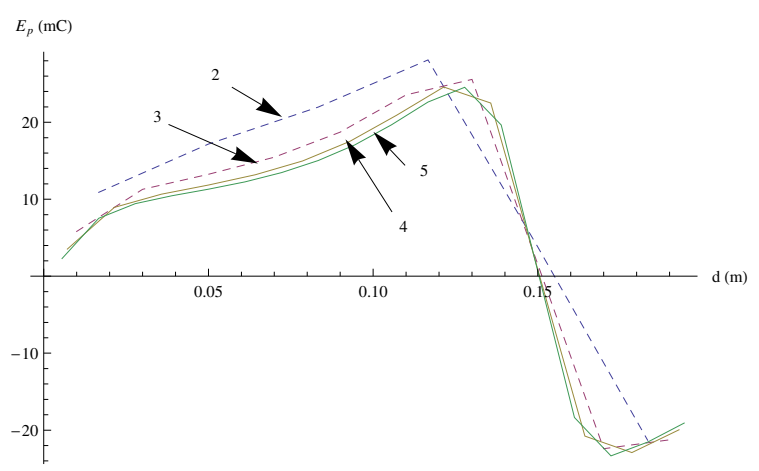

Figure 2: The polarization field distribution $E_{p}$ over the half plane between the transmission lines. Each of the curves represents a different Resolution, defined by Table 1 , for a constant volume sample.

\subsection{Operational Discretisation}

In the previous section we established that the discretisation level, labeled as Res 4 of Figure 2 and Table 1, provided the closest accessible level of mesh size that could represent the true environment. Computation at this level of discretisation is very slow however and certainly unacceptable for the real time applications required for the TDR. Even the precalculation of much of the Monte Carlo sampling described in [12] would be impractical at this level. It would therefore be advantageous to perform the calculations in the coarser mesh of Res 2 , and then use a map $Q$ to project the results into those that would have resulted from the finer mesh:

$$
Q: R^{h} \rightarrow R^{l}
$$

Where the high resolution (Res 4) and low resolution (Res 2) are represented by the subscripts $h$ and $l$ respectively. The observational Equation 23 for the high resolution, Res 4, can be written in this terminology as:

$$
T_{h}=F_{h}\left(\epsilon_{r}\right)+\mathcal{E}_{n}
$$

Where $t_{p}$ is the propagation time and $\mathcal{E}_{n}$ is the measurement noise. Applying the operator, $Q$, this becomes:

$$
T_{h}=Q F_{l}\left(\epsilon_{r}\right)+\mathcal{E}_{d}+\mathcal{E}_{n}
$$

with

$$
\mathcal{E}_{d}=\left(F_{h}\left(\epsilon_{r}\right)-Q F_{l}\left(\epsilon_{r}\right)\right)
$$

being the error introduced by using the coarser discretisation. In this sense the operator $Q$ can be termed as the model reduction operator [8]. Putting the total error as:

$$
\overline{\mathcal{E}}=\mathcal{E}_{d}+\mathcal{E}_{n}
$$

Equation 31 can be written as:

$$
T_{h}=Q F_{l}\left(\epsilon_{r}\right)+\overline{\mathcal{E}}
$$

Where we have made the assumption throughout this formulation that the discretisation error is approximately independent of the relative permittivity, $\epsilon_{r}$. This is discussed in more detail in Section 3.4.

With the propagation time measurement $T=t_{h}$ the form of the likelihood function becomes:

$$
\pi\left(t_{h} \mid \epsilon_{r}\right)=\pi_{\overline{\mathcal{E}}}\left(t_{h}-q f_{\ell}\left(\epsilon_{r}\right)\right)
$$

where the subscript $\overline{\mathcal{E}}$ emphasises that the likelihood distribution is of the same form as the distribution given by the combined error of Equation 33. If the $\pi_{\overline{\mathcal{E}}}$ is close to Gaussian, which is often the case, [9] shows that the above equation becomes:

$$
\begin{gathered}
\pi\left(t_{h} \mid \epsilon_{r}\right)=\exp \left[( t _ { h } - q f _ { \ell } ( \epsilon _ { r } ) - \tilde { e } ) ^ { T } \Gamma _ { e } ^ { - 1 } \left(t_{h}\right.\right. \\
\left.\left.-q f_{\ell}\left(\epsilon_{r}\right)-\tilde{e}\right)\right]
\end{gathered}
$$

Where $\tilde{e}$ and $\Gamma_{e}$ are the expectation and covariance respectively of the total error distribution, $\overline{\mathcal{E}}$. Here the mutual dependance of $\epsilon_{r}$ and the approximation 
error have been ignored, a simplification that will be addressed further in the next section.

The values of $f_{\ell}\left(\epsilon_{r}\right)$ can be pre-calculated because of the unique system geometry and operational constraints [12], so once the operator $q$ is known the calculation speed of Equation 35 is acceptable for real time application. The required posterior density is thus:

$$
\begin{gathered}
\pi\left(\epsilon_{r} \mid t_{h}\right)=\exp \left[( t _ { h } - q f _ { \ell } ( \epsilon _ { r } ) - \tilde { e } ) ^ { T } \Gamma _ { e } ^ { - 1 } \left(t_{h}\right.\right. \\
\left.\left.-q f_{\ell}\left(\epsilon_{r}\right)-\tilde{e}\right)\right] \pi_{\mathrm{pr}}\left(\epsilon_{r}\right)
\end{gathered}
$$

\subsection{Prior Densities}

As discussed in [12] for many cases of practical interest the moisture within the sample can be considered as being stratified in the $\mathrm{x}-\mathrm{z}$ plane. Apart from reducing the number of unknown permittivity values to be calculated, plane stratification also leads to symmetry in the $\mathrm{x}-\mathrm{z}$ plane that can be utilized to decrease the number of dimensions of the inverse system. In keeping with this stratified geometry of the sample we define three prior distributions for $\epsilon_{r}$, each representative of a number of real world scenarios. In the first case, the prior is given as a positive increase in relative permittivity with depth. With the discretisation level of Res 4 it can be defined as:

$$
\pi_{p r}\left(\epsilon_{r_{i+1}}\right)=\mathcal{U}\left[\epsilon_{r_{i}},+2\right]
$$

where $i=1 \ldots 6, \mathcal{U}$ is the uniform distribution and $\pi_{p r}\left(\epsilon_{r_{1}}\right)=\mathcal{U}[+2,+24]$.

The second prior is similarly defined but with a decrease in permittivity with depth, so that the incremental permittivity between layers is defined by $\mathcal{U}\left[\epsilon_{r_{i}},-2\right]$. For both of these distributions the maximum change of $\epsilon_{r}$ over the sample depth is 14 . A third prior is included as a subset of the first, that of a positive linear gradient over the sample. In this case the $\epsilon_{r}$ value of the upper boundary of the sample is chosen from the distribution $\pi\left(\epsilon_{r_{1}}\right)=\mathcal{U}[+2,+24]$, while the lower sample boundary value is chosen from $\pi\left(\epsilon_{r_{2}}\right)=\mathcal{U}\left[\epsilon_{r_{1}},+10\right] . \epsilon_{r}$ for the centre of each depth layer is then obtained by linear interpolation. Each of these will be used in the examples that follow.

\subsection{Mapping Operator}

After defining the form of $\pi_{p r}\left(\epsilon_{r}\right)$ the next immediate concern is to find a model reduction operator $Q$ that maximizes the decrease of $\mathcal{E}_{d}$ in Equation 32 and thus $\Gamma_{e}$ in Equation 37. For such an operator to be useful it must be only weakly dependant on the actual sample relative permittivity $\epsilon_{r}$.

Figure 3 is a plot of the propagation times, $t_{\ell}$ and $t_{h}$ when the sample is discretised to the level of Res 2 and Res 4 respectively, for 200 different simulations.
The values for each of the layer permittivities of Res 4 are generated by a prior distribution, $\mathcal{U}\left[\epsilon_{r_{i}},+2\right]$ defined in 3.3. Res 2 values for each case are calculated at the cell centre points of the three layers by Lagrange interpolation over the midpoints of the seven Res 4 cells. Figure 3 shows the relationship between the two propagation times for three transmission line heights above the sample. A good fit to the data for each height in Figure 3 is achieved by the polynomial:

$$
t_{h}=a+b\left(t_{\ell}\right)+c\left(t_{\ell}\right)^{-1}+d\left(t_{\ell}\right)^{-1 / 2}
$$

Where a, b, c and are chosen for each of the different heights. In terms of the Bayesian paradigm of Equation 37 then:

$$
q=a+b\left(t_{\ell}\right)+c\left(t_{\ell}\right)^{-1}+d\left(t_{\ell}\right)^{-1 / 2}
$$

Values of the coefficients for this polynomial are easily calculated by any of the standard fitting routines and are applicable to all measurements with the same geometrical relationships and prior relative permittivity distributions. The form of Equation 40 is also a valid approximation for the reduction operator when the other prior distributions defined in Section 3.3 are used, though the coefficients at similar heights may differ.

Table 2 shows the standard deviation, $\sigma_{d}$, of data difference from the fitted polynomial for the different priors discussed above. These values can be used for defining the distribution $\mathcal{E}_{d}$ in Equation 33 and together with the measurement noise, $\mathcal{E}_{n}$ constitute the total error, from which the covariance, $\Gamma_{e}$ of Equation 37 is extracted. The mean error in all fitted cases is zero and since $E\left\{\mathcal{E}_{n}\right\}=0$ we have $\tilde{e}=E\{\overline{\mathcal{E}}\}=0$. Further, the low dependance of the discretisation error on $\epsilon_{r}$ shown explicitly in Figure 3, strengthens the ability of the enhanced error model of Equation 37 to represent the real situation.

Using $\sigma_{d}$ from Table 2 and $\sigma_{n}=\mathcal{U}\left[0,10^{-12}\right]$ from previous experimental work [12], the Gaussian distributions $\mathcal{E}_{d}$ and $\mathcal{E}_{n}$ can be defined for Equation 33. The errors are dependant upon the current transmission line height only, so:

$$
\Gamma_{e_{\mathrm{ii}}}={\sigma_{d_{i}}}^{2}+{\sigma_{n_{i}}}^{2}
$$

is a diagonal matrix containing the total error variance for each height. Re-writing Equation 37 with $\tilde{e}=0$ and the covariance $\Gamma_{e_{\mathrm{ii}}}$ gives the form of the posterior density used in subsequent simulations as:

$$
\begin{array}{r}
\pi\left(\epsilon_{r} \mid t_{h}\right)=\exp \left[( t _ { h } - q f _ { \ell } ( \epsilon _ { r } ) ) ^ { T } \Gamma _ { e _ { i i } } ^ { - 1 } \left(t_{h}\right.\right. \\
\left.\left.-q f_{\ell}\left(\epsilon_{r}\right)\right)\right] \pi_{\mathrm{pr}}\left(\epsilon_{r}\right)
\end{array}
$$




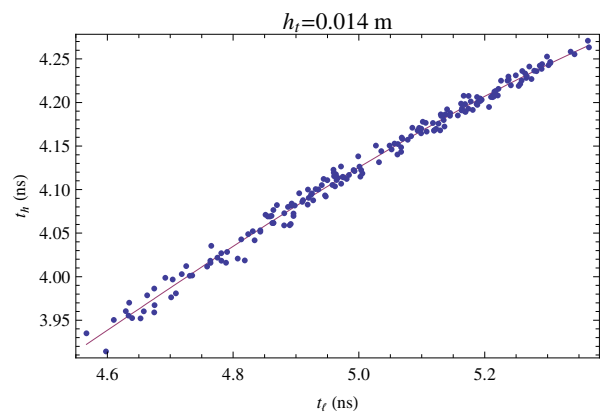

Table 2: The standard deviation, $\sigma_{d}$, of data fitted to Equation 40for the indicated transmission line heights . Given in ps $\left(10^{-12}\right.$ s $)$

\begin{tabular}{|l|lll|}
\hline$\pi_{p r}\left(\epsilon_{r}\right)$ & $h_{t}$ & $h_{t}$ & $h_{t}$ \\
& 0.006 & 0.01 & 0.014 \\
\hline $\mathcal{U}[0,+2]$ & 30.0 & 13.7 & 7.6 \\
$\mathcal{U}[0,-2]$ & 27.8 & 10.5 & 5.6 \\
linear +ve & 12.5 & 6.7 & 3.2 \\
\hline
\end{tabular}

Table 3: System parameters

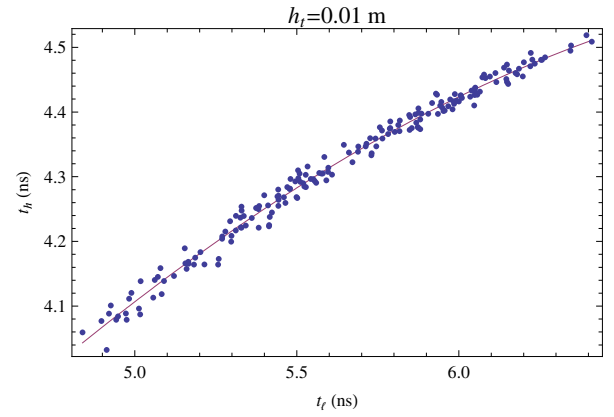

$h_{t}=0.006 \mathrm{~m}$

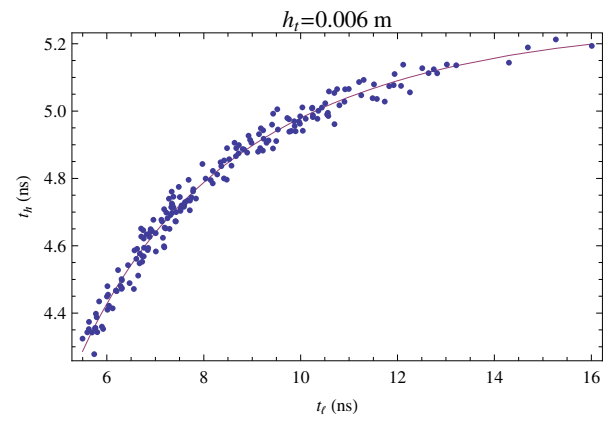

Figure 3: The form of the projection operator $Q$ between the low resolution and high resolution discretisation models for different transmission line heights $\left(h_{t}\right)$ above the sample. $t_{\ell}$ and $t_{h}$ are the propagation times for Res 2 and Res 4 respectively.

Thus the posterior distribution of $\pi\left(\epsilon_{r} \mid t_{p}\right)$ can be generated with the computationally accessible Res 2 . From this distribution an estimate of $\epsilon_{r}$ for each layer can made from the CM of Equation 26 with its associated error estimate given by the covariance defined by Equation 27.

sectionExample Having formulated a solution to the operational objectives outlined in the Introduction it remains to show how well it performs. Since this is a simulation, it is sensible to test the operational procedure by using the high resolution and computationally intensive (Res 4) simulated values to represent the "real world" instantiation of the TDR system and measurement.

Table 3 describes the physical dimensions of the transmission lines and their heights above the sample. These parameters remain constant throughout all simulations (including those of the previous section) in this paper.

\begin{tabular}{|l|l|}
\hline Parameter & Value \\
\hline Sample Depth & $0.1 \mathrm{~m}$ \\
Transmission Line & \\
$\quad$ Length & $\mathrm{d}=1$ \\
$\quad$ Diameter & $\mathrm{a}=\mathrm{a}=0.01$ \\
$\quad$ Separation & $\mathrm{b}=0.3) \mathrm{m}$ \\
Transmission Line & \\
Heights $\left(h_{m}\right)$ & $(0.006,0.01,0.014) \mathrm{m}$ \\
\hline
\end{tabular}

\subsection{Relative Permittivity Profiles}

Random values of $\epsilon_{r}$ were assigned to each one of the 7 depth layers defined by Res 4 , according to the prior scenarios depicted in Table 2. Using Equation 4 , the simulated (or measured) value for $t_{h}$ was then calculated at each transmission line height. Using the appropriate value for the reduction operator, $q$, and the Monte Carlo procedure detailed in [12] for the lower resolution $f_{\ell}\left(\epsilon_{r}\right)$, the posterior density of Equation 42 was generated. An estimate for $\epsilon_{r}$ was then determined from the CM of Equation 27 and compared with the original $\epsilon_{r}$ values. Since Res 4 has 7 layers its profile is linearly interpolated to the cell centres of the Res 2 case for this comparison. Table 4 shows the standard deviation of the difference between the simulated and estimated value of $\epsilon_{r}$ for 200 cases of each prior scenario. The standard deviation for each case is small compared to the range of $\epsilon_{r}$ covered and is well within requirements for practical use, where volumetric moisture content is to be estimated (next section). Note that there is a small decrease in the error as the prior distribution is more tightly constrained (i.e. between the more flexible uniform distribution profiles and the constrained linear profile).

Note, because $\overline{\mathcal{E}}$ is Gaussian with mean $\tilde{e}=0$ and a large number of samples are used, one can either use the form of the likelihood function in Equation 42 or the simplified version taking into account only the measurement noise variance $\left(\gamma_{n}\right)$, $\exp \left[\gamma^{-1}\left\|t_{h}-q f_{\ell}\left(\epsilon_{r}\right)\right\|^{2}\right]$ to obtain the statistics on the error in determining $\epsilon_{r}$. In the following, the form of Equation 42 is used since it is the most general since estimates of error can be obtained for a single sample.

\subsection{Moisture Profiles}

The object of the TDR method is to measure the moisture content profile of a sample with depth. The inverse methodology discussed previously has focused 
Table 4: Standard Deviation, $\sigma_{\epsilon}$, of the difference between the estimated and simulated values of $\epsilon_{r}$.

\begin{tabular}{|l|llll|}
\hline Layer & $\begin{array}{l}\text { Depth } \\
(\mathrm{m})\end{array}$ & $\mathcal{U}[0,+2]$ & $\mathcal{U}[0,-2]$ & $\begin{array}{l}\text { linear } \\
\text { +ve }\end{array}$ \\
\hline 1 & 0.016 & 0.46 & 0.43 & 0.21 \\
2 & 0.050 & 0.45 & 0.46 & 0.34 \\
3 & 0.066 & 0.74 & 0.65 & 0.65 \\
\hline
\end{tabular}

upon relative permittivity since the mapping from this to a moisture estimate is straightforward and can be applied after the inversion procedure. Topp [3] showed that this mapping could be described by:

$$
\begin{aligned}
\theta_{v}=- & 5.310^{-2}+2.9210^{-2} \epsilon_{r} \\
- & 5.510^{-4} \epsilon_{r}^{2}+4.310^{-6} \epsilon_{r}^{3}
\end{aligned}
$$

Where $\theta_{v}$ is the volumetric moisture content.

As a realistic example of an application for which the TDR is intended, consider the moisture profile of a sample of unsaturated loamy soil. Under typical conditions, the moisture content in the sample increases with depth as the sun dries out the top layer during the day. Figure 4 represents such a situation with some added complexity in the mid depth simulating soil material variation. Typical moisture profile variation over a depth of $0.1 \mathrm{~m}$ can be anywhere between $0 \%$ to $25 \%$, (e.g. [14]) so this example is close to the maximum variation expected. From the original profile, $\theta_{v}$ values corresponding to the cell centres of the fine mesh are taken and converted to the corresponding $\epsilon_{r}$ via interpolation of Equation 43. These values are then used for the Res 4 permittivity profile to generate the simulated observations, $t_{h}$, after which the method described in the previous section is used to estimate the value of $\epsilon_{r}$ with the reduced system calculations. The estimates, returned to volumetric moisture via Equation 43, are also shown in Figure 4. A second example is given in Figure 5 where the moisture content nears field capacity at the surface and dries out with depth, a situation during heavy rain. Both examples show a good reconstruction of the moisture profile, albeit at the lower depth resolution corresponding to the 3 layer discretisation of Res 2 . Also shown on both figures are the MAP estimates of $\theta_{v}$ for the three coarse mesh layers, using the same Monte Carlo data. Their poor ability to reconstruct the simulated curve indicates that a minimization procedure would require further real time processing (e.g. extended distribution sampling using some form Markov chain Monte Carlo method). There seems little to be gained from pursuing this path at present.

In more general terms than the examples above, the expected error in volumetric moisture content, $\theta_{v}$, can be derived from applying $\delta \epsilon_{r}=2 \times \sigma_{d}$ of Table 4 to the differential of Equation 43:

$$
\delta \theta_{v}=\left(0.0292-0.0011 \epsilon_{r}+0.0000129 \epsilon_{r}^{2}\right) \delta \epsilon_{r}
$$

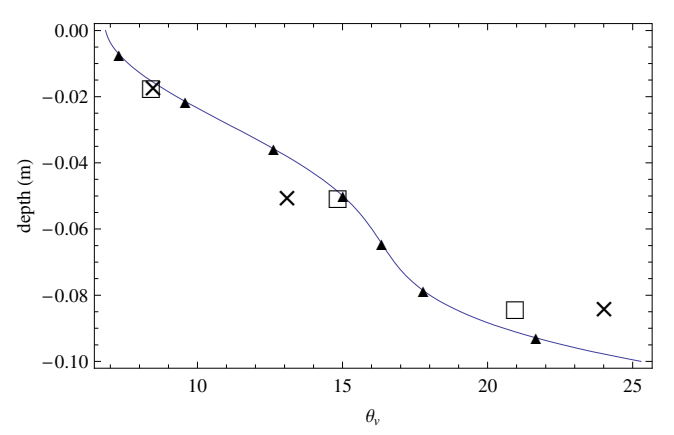

Figure 4: Simulated depth profile for the percentage volumetric moisture content, $\theta_{v}$ of a soil sample. The form of the moisture distribution with depth follows the prior distribution of permittivity given by $\mathcal{U}[0,+2]$ between layers. Physically this corresponds to a loamy soil drying near the surface. The solid line is the original model for the moisture content depth profile, while the $\boldsymbol{\Delta}$ are the points chosen for the midpoint of the 7 layer Res4 representation. The CM and MAP estimates of the inverse in Res 2 are shown as $\square$ and $\times$ respectively .

In more general terms than the examples above, the expected error in volumetric moisture content, $\theta_{v}$, can be derived from applying $\delta \epsilon_{r}=2 \times \sigma_{d}$ of Table 4 to the differential of Equation 43:

$$
\delta \theta_{v}=\left(0.0292-0.0011 \epsilon_{r}+0.0000129 \epsilon_{r}^{2}\right) \delta \epsilon_{r}
$$

Figure 6 shows the expected error in percentage $\theta_{v}$ for the two relative permittivity priors defined by $\mathcal{U}[0,-2]$ and $\mathcal{U}[0,+2]$. Errors for all depths are less than 4.0 $\%$ and this indicates excellent resolution compared to invasive techniques,(e.g. [15] [16]).

\section{Conclusion and Discussion}

The aim of this work has been to simplify the TDR inversion model so that its real time application can be achieved. To this end we have decomposed the original model into a reduced set of cells by exploiting both planar geometry and system symmetry. This together with the likely distribution of moisture content with depth provided a powerful prior model for the inverse process defined in a Bayesian framework. The level of discretisation required to avoid errors through the discretisation itself has been determined to be 


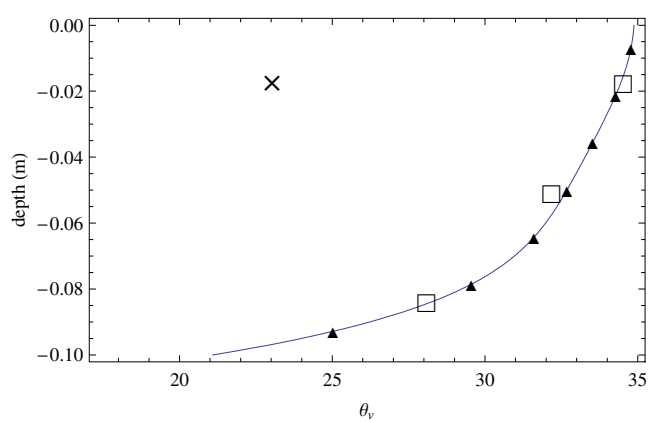

Figure 5: Similar to Figure 4 but with a permittivity prior of $\mathcal{U}[0,-2]$ between the layers. The physical situation represents a moisture content near field capacity at the surface and drying with depth. The CM estimate is shown by $\square$. Note that only one MAP estimate $(x)$ is within the plot range.

given by a cell size of $0.014 \mathrm{~m}$ for the proposed operational system geometry. Since this level of discretisation is impractical for operational use, an effective reduction operator has been developed that allows the TDR inversion procedure to be carried out at a much coarser mesh level (a cell size of 0.0333 $\mathrm{m})$. Further, the use of this reduction operator produces a relatively small error in the CM estimate of the relative permittivity in the layered sample. With the Monte Carlo approach previously developed [12], the inversion is easily accommodated within a time scale and accuracy suitable for "in the field" use of the TDR equipment. As pointed out, many practical samples do not have a rapidly changing permittivity (or direction of gradient) over the $100 \mathrm{~mm}$ or so of depth, so for many practical purposes the prior scenarios studied are appropriate.

In practice there are a number of considerations to be assessed when applying the procedure described here to an operational system. One such consideration is the dependance of the reduction operator on the moisture (or relative permittivity) profile of the sample. While it is clear that coefficients for the parameterising curve given by Equation 40 are dependant upon the height of the transmission lines above the sample, less clear is how they vary with the sign of gradient of $\epsilon_{r}$. For example Figure 7 shows the polynomial fit to Equation 40 for the three prior scenarios reviewed. Clearly the uniform non linear and linear profiles for positive gradients are very close. Indeed the linear positive prior is really a subset of the more general uniform non-linear case. On the other hand the uniform non linear negative gradient is substantially different and an attempt to represent its reduction operator by either of the other two would cause a significant additional error. This suggests that two sets of coefficients only for each height may be necessary for complete specification and this needs further investigation.

In many cases the correct gradient direction can be inferred from the type of sample. When this is not the case however, it would be advantageous to be able to
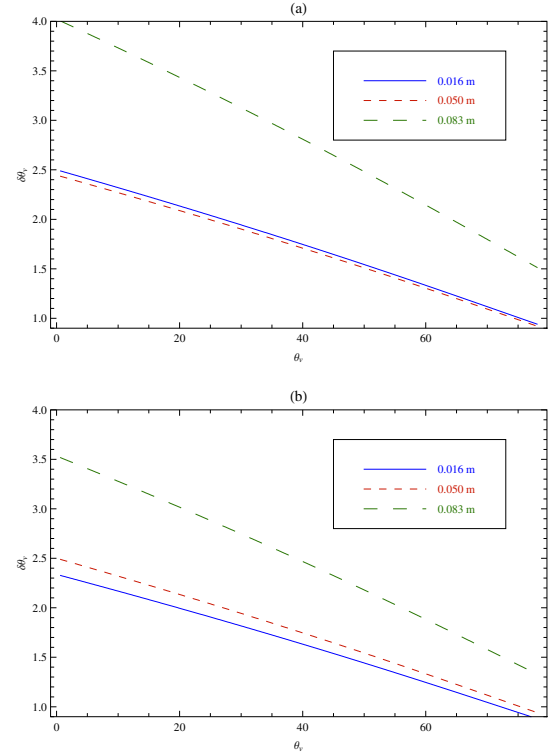

Figure 6: The estimated error $\left(\delta \theta_{v}\right)$ in $\theta_{v}$ for the indicated depths below the surface of the sample for

(a) prior $\mathcal{U}[0,-2]$ and (b) prior $\mathcal{U}[0,+2]$

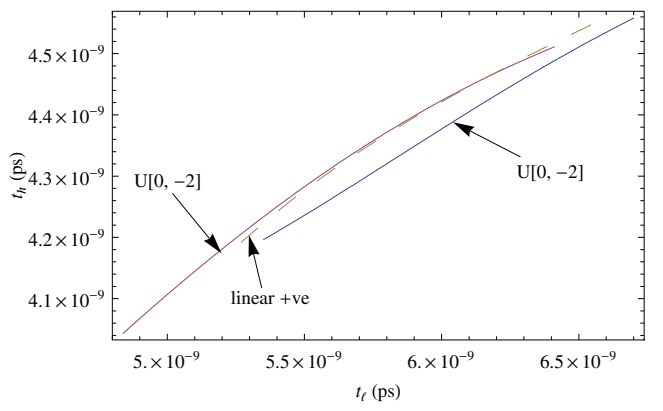

Figure 7: Polynomial fit of Equation 40 for the three prior scenarios described in 3.3 with $h_{t}=0.01 \mathrm{~m}$. The two scenarios with positive $\epsilon_{r}$ gradient are similar,

while that with a negative gradient $\mathcal{U}[0,-2]$ is markedly different.

determine this as part of the measurement procedure so that the correct coefficients for Equation 40 can be applied. Alternatively, it is possible that a good indication of when the wrong reduction operator was chosen can be gleaned from the values of the posterior distribution. Both of these measures are the subject of current work.

A second consideration is improving the electric field distribution representation within the sample. [13] showed that an improvement over the cell centre point matching method, conventionally used in integration by moment methods, can be accomplished by adding a proximity compensation in each of the cells adjacent to the one under consideration. Such an approach can further counterpoise the discretisation process and has yet to be included in the operational TDR system. 


\section{References}

[1] K. Noborio, Measurement of soil water content and electrical conductivity by time domain reflectometry: a review, Computers and Electronics in Agriculture, 31, 2001, 213-237.

[2] S. R. Evett and G. W. Parkin, Advances in Soil Water Content Sensing: The Continuing Maturation of Technology and Theory, Vadose Zone J, 4, 2005, 986-991.

[3] G. Topp, State of the art measuring soil water content, Hydrological Processes 17 (2003), 29932996.

[4] J. Ledieu, P. De Ridder, P. De Clerck and S. Dautrbande, A method of measuring soil moisture by time domain reflectometry, Journal of Hydrology 88 (1986), 319-328.

[5] I. M. Woodhead, I. G. Platt, Pseudo-Static Electromagnetic Modelling of Moisture Sensors in Lossy Dielectrics, Seventh Conference on Electromagnetic Wave Interaction with Water and Moist Substances, Hamamatsu, Japan, 2007.

[6] I. M. Woodhead, G. Buchan and D. Kulasiri, Pseudo 3-D Moment Method for Rapid Calculation of Electric Field Distribution in a Low Loss Inhomogeneous Dielectric, IEEE Transactions on Antennas and Propagation 49 (2001), 1117-1122.

[7] I. M. Woodhead, G. Buchan, D. Kulasiri and J. Christie, A new model for the response of TDR to heterogenous dielectrics, Subsurface Sensing Technologies and Applications 1 (2000), 473487.

[8] J. Kaipio and E. Somersalo, Statistical and Computational Inverse Problems, SpringerVerlag, New York, 2005.

[9] S. R. Arridge, et al., Approximation errors and model reduction with an application in optical diffusion tomography, Inverse Problems 22 (2006), 175-195.

[10] R. F. Harrington, Field Computation by Moment Methods, R. E. Kreiger, 1987.

[11] W. C. Elmore and M. A. Heald, Physics of Waves, McGraw-Hill, NY, 1969.

[12] I. G. Platt and I. M. Woodhead, A 1-D Inversion for Non-Invasive Time Domain Reflectometry, To be Published in Measurement Science and Technology, 2008.

[13] I. Woodhead, G. Buchan, I. Platt and J. Christie, Improved Modelling for TDR, Meas. Sci. Technol. 18 (2007), 1110-1117.

[14] R. G. McLaren and K. C. Cameron, Soil Science, Oxford University Press, 1990.
[15] D. A. Robinson and S. B. Jones and J. M. Wraith and D. Or, and S. P. Friedman, A Review of Advances in Dielectric and Electrical Conductivity Measurement in Soils Using Time Domain Reflectometry, Vadose Zone J, 2, 2003, 444-475.

[16] Magnus Persson and Sahar Haridy, Estimating Water Content from Electrical Conductivity Measurements with Short Time-Domain Reflectometry Probes, Soil Sci Soc Am J, 67, 2003, 478-482. 